\section{Serviço de Atendimento Móvel de Urgência (SAMU): análise da demanda e sua distribuição espacial em uma cidade do Nordeste brasileiro}

\section{Mobile Emergency Care Service (SAMU): analysis of demand and its space distribution in a city of the Brazilian northeast}

\section{Amanda Priscila de Santana Cabral Wayner Vieira de Souza}

Departamento de Saúde Coletiva - NESC do Centro de Pesquisa Aggeu Magalhães / Fundação Oswaldo Cruz (CPqAM/FIOCRUZ)

Correspondência: Amanda Priscila de Santana Cabral, Rua Ribeiro de Brito, 930/201 - Boa Viagem, 51021-310 - Recife, PE

E-mail: amandacabral@yahoo.com.br

\section{Resumo}

O município de Olinda, Pernambuco, criou instrumento para armazenamento de dados dos formulários das ocorrências do Serviço de Atendimento Móvel de Urgência (SAMU-192) e de forma pioneira georreferencia os atendimentos realizados.

Objetiva-se descrever o perfil epidemiológico das ocorrências atendidas no município, de fevereiro (implantação do serviço) a junho de 2006, com ênfase na distribuição espacial das ocorrências mais relevantes. Foram utilizados dados secundários do banco de dados SAMU-192, considerando a freqüência das seguintes variáveis: sexo, faixa etária, tipo de ocorrências, dias da semana, tipo de causas clínicas, tipo de causas externas, tipo de acidentes de transportes e veículos envolvidos. Tendo por base as freqüências dos tipos de causas clínicas/ externas, identificaram-se aquelas de maior magnitude para mapeamento e identificação dos aglomerados espaciais com o emprego de estimador de intensidade Kernel.

Das 1956 ocorrências, 1114 foram por causas clínicas e 645 por causas externas; finais de semana acumularam $46,0 \%$ dos atendimentos; $55,1 \%$ das ocorrências por causas clínicas foram em mulheres, enquanto $72,1 \%$ das causas externas em homens. A média etária para as causas clínicas foi de 47 anos e 34 anos para causas externas. Destacaram-se as doenças do aparelho circulatório (23,1\% das causas clínicas) e acidentes de transporte (52,7\% das causas externas); desses $61,1 \%$ motivados por atropelamentos e $33,6 \%$ com motocicletas envolvidas.

A análise espacial reforça a necessidade da integração entre a Secretaria de Saúde e órgãos afins para a implantação de medidas preventivas, e o perfil epidemiológico apresentou informações capazes de auxiliar na organização do serviço e na compreensão do perfil de morbidade.

Palavras-chave: Atendimento de Emergência Pré-Hospitalar. Perfil epidemiológico. Medicina de urgência. Distribuição espacial. 


\section{Abstract}

The city of Olinda (Pernambuco, Brazil) created a tool for storing data from the Emergency Mobile Healthcare Service forms (SAMU-192) which is a pioneer in providing georeferenced information on the treatment given.

The aim of the present study was to describe the epidemiological profile of care in the city from February (implementation of the service) to June 2006, with an emphasis on the spatial distribution of the most relevant events. Secondary data from the SAMU-192 database was used, considering the frequency of the following variables: gender, age, type of event, day of the week, type of clinical cause, type of external cause, type of traffic accident and vehicles involved. Based on the frequency of the types of clinical/ external causes, those of greater magnitude were used for mapping and identifying spatial clusters using the Kernel intensity estimator.

Among the 1,956 events, 1,114 were due to clinical causes and 645 were due to external causes; weekends totaled $46.0 \%$ of the events; $55.1 \%$ of events due to clinical causes were in women, whereas $72.1 \%$ of external causes were in men. The average age for clinical causes was 47 years and for external causes was 34 years. Circulatory system diseases accounted for $23.1 \%$ of clinical causes. Traffic accidents accounted for $52.7 \%$ of external causes, $61.1 \%$ of which were run overs and $33.6 \%$ involved motorcycles.

The spatial analysis emphasized the need for integration between the Health Department and other agencies in order to implement preventive measures. The epidemiological profile offers information that can assist in the organization of the service and in the understanding of the morbidity profile.

Keywords: Pre-Hospital Emergency Care. Epidemiological profile. Emergency medicine. Spatial distribution.

\section{Introdução}

Historicamente, o nível de resposta do sistema de saúde às urgências $\mathrm{e}$ emergências é insuficiente, provocando a superlotação das portas dos hospitais e pronto-socorros, mesmo quando a doença ou quadro clínico não é característica de um atendimento de emergência ou urgência ${ }^{1}$. Com a modificação do perfil epidemiológico da morbi-mortalidade devido ao crescimento das causas externas, esses atendimentos ganham maior relevância, causando forte impacto ao setor saúde e sua resposta a tal demanda é fundamental para minimizar as seqüelas decorrentes desse quadro ${ }^{2}$.

Em 29 de setembro de 2003 entraram em vigor duas importantes portarias: a 1863 GM, que institui a Política Nacional de Atenção às Urgências, a qual tem como um de seus componentes o atendimento pré-hospitalar móvel ${ }^{3}$, enquanto a segunda portaria, a $1864 \mathrm{GM}$, oficializa a implantação do Serviço de Atendimento Móvel de Urgência (SAMU-192) em municípios e regiões de todo o território brasileiro ${ }^{4}$.

O SAMU-192 destina-se ao atendimento de urgência e emergência nas residências, locais de trabalho e vias públicas. O socorro é feito após chamada gratuita para o telefone 192. A ligação é atendida por técnicos na central de regulação que imediatamente transferem o telefonema para o médico regulador. Esse profissional faz o diagnóstico da situação e inicia o atendimento no mesmo instante, orientando o paciente ou a pessoa que fez a chamada sobre as primeiras ações 5 .

Em 2005, o SAMU-192 funcionava em 784 municípios brasileiros de 25 Estados, com 101 centrais de regulação, atuando com equipes especializadas, em sintonia com centrais de controle de leitos nos hospitais ${ }^{6}$. Seu atendimento pode ser do tipo primário, quando oriundo do cidadão, ou do tipo secundário, também conhecido como remoção, quando a solicitação parte do serviço de saúde onde o paciente já tenha recebido os primeiros cuidados para estabilização do quadro de urgência ou emergência, mas 
necessite ser conduzido a outro serviço de maior complexidade para a continuidade do tratamento ${ }^{1}$.

Recomenda-se que os dados de urgência e emergência sejam utilizados para elaboração de uma linha de base descritiva dos serviços de saúde e dos perfis epidemiológicos existentes ${ }^{4}$. Entretanto, não há um instrumento oficial para armazenamento de informações das ocorrências atendidas.

Por outro lado, a gestão do sistema de saúde tem valorizado a análise de dados espaciais por aportar novos subsídios para o planejamento e avaliação das ações, baseados na análise da distribuição espacial das doenças, da localização dos serviços de saúde e dos riscos ambientais?.

O SAMU-192 foi implantado no município de Olinda, Pernambuco, em fevereiro de 2006. Conta com médicos, enfermeiros, técnicos de enfermagem e condutores que se distribuem em 04 ambulâncias de suporte básico e 01 ambulância de suporte avançado.

A secretaria municipal de saúde criou de forma pioneira um banco de dados no software EpiInfo 3.3. $2^{8}$, com dados dos formulários de atendimentos, para possibilitar a análise epidemiológica das ocorrências. Outro diferencial do atendimento pré-hospitalar móvel do município é o fato de todos os atendimentos serem georreferenciados, uma vez que suas ambulâncias apresentam o aparelho Global Positioning System (GPS) e os condutores foram treinados em seu manuseio.

Por se tratar de uma estratégia recente e pela ausência de instrumento de captação de dados em âmbito nacional, existe uma carência de estudos que reflitam a demanda do SAMU-192. Este trabalho procura descrever o perfil epidemiológico das ocorrências atendidas pelo serviço em Olinda, no período que abrange a implantação em fevereiro até junho de 2006, com ênfase na distribuição espacial das ocorrências de maior magnitude, objetivando contribuir para o monitoramento das urgências e emergências e, conseqüentemente, favorecer a integração intersetorial no município.

\section{Métodos}

A área de estudo é o município de Olinda, localizado na região metropolitana de Recife, Pernambuco. Segundo projeção do DATASUS ${ }^{9}$ para o ano de 2006, este município conta com 387.496 habitantes, distribuídos num território de 40,83 $\mathrm{Km}^{2} \mathrm{e}$ densidade demográfica de 9.490,45 Hab./ $\mathrm{Km}^{2}$, a quinta maior do país ${ }^{10}$

Este é um estudo descritivo, com utilização de dados secundários do banco de dados do SAMU-192 Olinda, referentes ao total de ocorrências atendidas de 24 de fevereiro a 30 de junho de 2006, período de implantação do serviço.

Para a análise do perfil epidemiológico dos atendimentos, calculou-se a partir das variáveis sexo e faixa etária, as freqüências relativas e os coeficientes de incidência (incidência cumulativa). Para tanto, tomou-se a população estimada pelo IBGE para 2006, sendo 181.430 habitantes do sexo masculino e 206.066 do sexo feminino e, segundo faixa etária, 66.574 habitantes menores de 10 anos, 76.377 habitantes de 10 a 19 anos, 134.703 habitantes de 20 a 39 anos, 75.253 habitantes de 40 a 59 anos e 34.589 habitantes de 60 anos e mais.

Para as variáveis tipo de ocorrência, dia da semana, tipo de causa clínica, tipo de causa externa, tipo de acidente de transporte e veículo envolvido, foram calculadas as freqüências relativas.

Baseada na distribuição de freqüência das variáveis "tipo de causa clínica" e "tipo de causa externa” foram identificadas aquelas de maior volume de ocorrências para realização da distribuição espacial. Os dados georreferenciados foram lançados no aplicativo TerraView 3.1.3 ${ }^{11}$ para o mapeamento e a identificação dos aglomerados espaciais, com o emprego de estimador de intensidade Kernel.

O método de alisamento por função Kernel único é técnica não paramétrica que promove o alisamento - ou suavização estatística -, o que permite filtrar a variabilidade de um conjunto de dados, retendo as 
características essenciais locais dos dados. Desse modo, faz-se a estimativa alisada da intensidade local dos eventos sobre a área estudada, obtendo-se uma "superfície de risco" para sua ocorrência ${ }^{12}$. A estimativa básica para a intensidade do padrão de pontos na posição s é:

$\hat{\lambda}_{\tau}(s)=\sum_{i=1}^{n} \frac{1}{\tau^{2}} k\left(\frac{\left(s-s_{i}\right)}{\tau}\right)$ onde:

$k$ ( ) - função de alisamento gaussiano;

$\tau$ - é a largura da banda que define o grau

de alisamento;

$\mathrm{s}$ - centro da área a ser estimada;

si - localização dos eventos;

$n$-número total de pontos (eventos);

$\lambda^{\wedge} \tau(s)$ - é o valor estimado.

Para cada $\mathrm{k}($ ) escolhido e banda $\tau, o$ $\lambda$ (s) é estimado em cada ponto na região R. Desta forma, obtém-se a estimativa da densidade de eventos ocorridos por unidade de área, a qual é atribuída às células componentes de uma grade regular que abrange a região estudada ${ }^{12}$.

O estimador de intensidade Kernel é um indicador de fácil uso e interpretação; entretanto, não considera a distribuição populacional onde os eventos estão inseridos. Um método capaz de reverter esta desvantagem é a utilização do estimador de Kernel num dado de incidência de doença ou evento, já que a incidência por si só considera a população de risco ou exposta a tal agravo ${ }^{13}$.

Para as causas externas das quais a simples localização do evento é objeto de análise, utilizou-se o Kernel único, enquanto nos casos em que foi necessário o conhecimento da base populacional, como nas causas clínicas, foi aplicado o Kernel de incidência, possibilitando identificar a concentração das maiores taxas de demanda para este agrupamento de doenças.

Este estudo foi submetido e aprovado pelo Comitê de Ética em Pesquisa do Centro de Pesquisas Aggeu Magalhães / Fiocruz sob registro no CAEE No 0061.0.095.000-06.

\section{Resultados}

Foram atendidas 1.956 ocorrências, sendo $1.114(57,0 \%)$ por causas clínicas, 645 $(32,9 \%)$ por causas externas e $143(7,3 \%)$ por remoções. Em 54 (2,8\%) formulários de ocorrências, esse dado foi ignorado. Os finais de semana acumularam $46 \%$ dos atendimentos e, apesar do predomínio de ocorrências por causas clínicas de segunda feira a quinta feira, nos finais de semana foi observado um aumento do número absoluto de ocorrências por causas externas (Figura 1).

A média etária do total de ocorrências foi de 43 anos e a mediana de 39 anos, sendo notadas particularidades, segundo tipos de causa, enquanto as ocorrências por causas clínicas tiveram uma média de 47 anos e uma mediana de 46 anos, observou-se uma queda da idade nos atendimentos por causas externas, sendo a média de 34 anos e a mediana de 31 anos $(\mathrm{p}<0,001)$.

A Tabela 1 aponta que a faixa de 20 - 39 anos concentrou a maior proporção do total de atendimentos $(33,7 \%)$ e causas externas (48,1\%); os idosos apresentaram a maior incidência de atendimentos (126/10.000 habitantes) e participaram de cerca de um terço das causas clínicas; do total de atendimentos, $6,2 \%$ foram destinados aos menores de 10 anos, dos quais 8,7/10.000 habitantes foram por causas clínicas e 4,2/10.000 habitantes por causas externas (Tabela 1 ).

No total de ocorrências, $54,0 \%$ dos atendimentos destinaram-se ao sexo masculino (Tabela 2). Entretanto, a estratificação por tipo de causas mostra uma inversão nesta proporção nos atendimentos por causas clínicas, onde $55,1 \%$ se deram no sexo feminino, e revela que os homens apresentaram risco três vezes maior do que as mulheres de gerar ocorrências por causas externas (RR=2,95; IC $(2,48 ; 3,52)$ ) (Tabela 2).

A Tabela 3 mostra que $58,7 \%$ das ocorrências por causas clínicas se enquadram em três principais grupos de causas: doenças do aparelho circulatório, com $23,1 \%$ (sendo as doenças hipertensivas responsáveis por $71,5 \%$ das ocorrências deste grupo), sinto- 


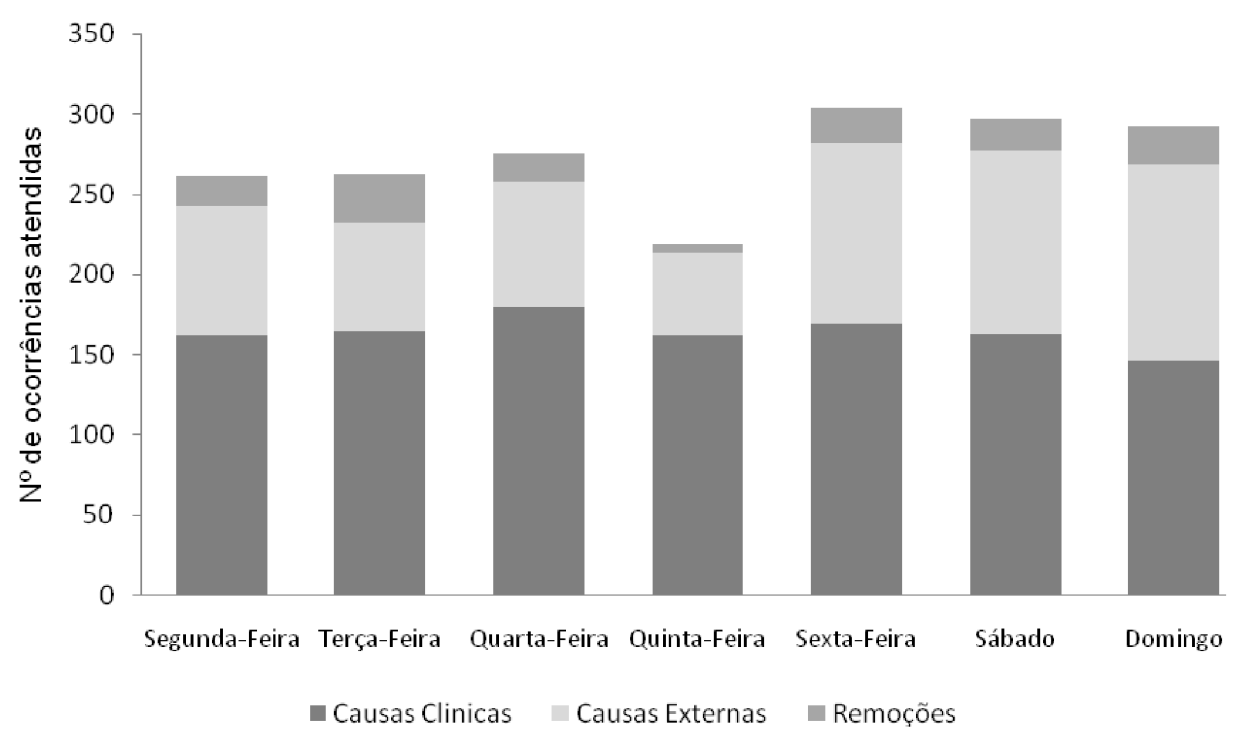

Figura 1 - Ocorrências atendidas pelo SAMU-192-Olinda segundo dia da semana e grupos de ocorrências. Olinda, PE - fevereiro a junho de 2006.

Fonte: SAMU-192-Olinda, 2006

Figure 1 - Events treated by SAMU-192/Olinda, according to day of the week and groups of events. Olinda, PE - February to June 2006.

Source: SAMU-192-Olinda, 2006

Tabela 1 - Tipos de ocorrências atendidas pelo SAMU - 192 - Olinda segundo faixa etária. Olinda, PE - fevereiro a junho de 2006.

Table 1 - Types of events treated by SAMU-192/Olinda according to age group. Olinda, PE - February to June 2006.

\begin{tabular}{lccccccccccccccc}
\hline \multirow{2}{*}{ Faixa etária } & \multicolumn{3}{c}{ Causa Clinica } & \multicolumn{3}{c}{ Causa Externa } & \multicolumn{3}{c}{ Remoções } & \multicolumn{3}{c}{ Ignorados } & \multicolumn{3}{c}{ Total } \\
& $\mathrm{N}$ & $\%$ & $\mathrm{Cl}$ & $\mathrm{N}$ & $\%$ & $\mathrm{Cl}$ & $\mathrm{N}$ & $\%$ & $\mathrm{Cl}$ & $\mathrm{N}$ & $\%$ & $\mathrm{Cl}$ & $\mathrm{N}$ & $\%$ & $\mathrm{Cl}$ \\
\hline$<10$ anos & 58 & 5,2 & 8,7 & 28 & 5,2 & 4,2 & 13 & 10,9 & - & 3 & 13,0 & - & 102 & 6,2 & 15,3 \\
$10-19$ anos & 85 & 7,6 & 11,1 & 69 & 12,8 & 9,0 & 16 & 13,4 & - & 2 & 8,7 & - & 172 & 10,5 & 22,5 \\
$20-39$ anos & 258 & 23,2 & 19,2 & 259 & 48,1 & 19,2 & 35 & 29,4 & - & 0 & 0,0 & - & 552 & 33,7 & 41,0 \\
$40-59$ anos & 228 & 20,5 & 30,3 & 122 & 22,7 & 16,2 & 25 & 21,0 & - & 2 & 8,7 & - & 377 & 23,0 & 50,1 \\
60 anos e mais & 330 & 29,6 & 95,4 & 60 & 11,2 & 17,4 & 30 & 25,2 & - & 16 & 69,6 & - & 436 & 26,6 & 126,1 \\
Subtotal & 959 & 100,0 & 28,8 & 538 & 100,0 & 13,9 & 119 & 100,0 & - & 23 & 100,0 & - & 1639 & 100,0 & 42,3 \\
lgnorados & 155 & - & - & 107 & - & - & 24 & - & - & 31 & - & - & 317 & - & - \\
Total & 1114 & - & - & 645 & - & - & 143 & - & - & 54 & - & - & 1956 & - & - \\
\hline
\end{tabular}

Fonte: SAMU-192-Olinda, 2006

Obs: $\mathrm{Cl}=$ Coef. De Incidência / 10.000 hab

Source: SAMU-192-Olinda, 2006

Obs: $\mathrm{Cl}=$ Incidence Coefficients $/ 10,000$ inhabitants

mas, sinais e achados anormais de exames clínicos e de laboratório, não classificados em outra parte $(21,8 \%)$, e gravidez, parto e puerpério (13,8\%). Destaca-se o fato de $22,4 \%$ dos atendimentos por causas clínicas terem motivação ignorada.

Os acidentes de transporte abarcaram $52,7 \%$ das ocorrências por causas externas
(Tabela 3). Os atropelamentos foram responsáveis $61,1 \%$ dos acidentes de transporte, enquanto as colisões por $26,1 \%$, seguidos por $8,9 \%$ de quedas no trânsito e $3,9 \%$ de capotamentos. O motivo da ocorrência por causas externas foi ignorado em cerca de $18,0 \%$ dos registros, no período estudado. 
Tabela 2 - Tipos de ocorrências atendidas pelo SAMU - 192 - Olinda segundo sexo. Olinda, PE - fevereiro a junho de 2006.

Table 2 - Types of events treated by SAMU-192/Olinda according to gender. Olinda, PE - February to June 2006.

\begin{tabular}{lccccccccccccccc}
\hline \multirow{2}{*}{ Sexo } & \multicolumn{3}{c}{ Causa Clinica } & \multicolumn{3}{c}{ Causa Externa } & \multicolumn{3}{c}{ Remoções } & \multicolumn{3}{c}{ Ignorados } & \multicolumn{3}{c}{ Total } \\
& $\mathrm{N}$ & $\%$ & $\mathrm{Cl}$ & $\mathrm{N}$ & $\%$ & $\mathrm{Cl}$ & $\mathrm{N}$ & $\%$ & $\mathrm{Cl}$ & $\mathrm{N}$ & $\%$ & $\mathrm{Cl}$ & $\mathrm{N}$ & $\%$ & $\mathrm{Cl}$ \\
\hline Masculino & 491 & 44,9 & 27,1 & 449 & 72,2 & 24,8 & 71 & 52,21 & - & 12 & 28,57 & - & 1023 & 54,0 & 49,6 \\
Feminino & 603 & 55,1 & 29,3 & 173 & 27,8 & 8,4 & 65 & 47,79 & - & 30 & 71,43 & - & 871 & 46,0 & 42,3 \\
Subtotal & 1094 & 100,0 & 28,2 & 622 & 100,0 & 16,0 & 136 & 100,0 & - & 42 & 100,0 & - & 1894 & 100,0 & 48,9 \\
Ignorados & 20 & - & - & 23 & - & - & 7 & - & - & 12 & - & - & 62 & - & - \\
Total & 1114 & - & - & 645 & - & - & 143 & - & - & 54 & - & - & 1956 & - & - \\
\hline
\end{tabular}

Fonte: SAMU-192-Olinda, 2006

Obs: $\mathrm{Cl}=$ Coef. De Incidência / 10.000 hab

Source: SAMU-192-Olinda, 2006

Obs: $\mathrm{Cl}=$ Incidence Coefficients $/ 10,000$ inhabitants

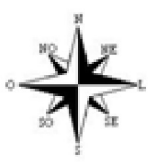

DENSIDADE DE OCORRÊNCIAS

Baixa Densidade

Média Densidade

Alta Densidade

- ESTABELECIMENTOS DE SAĹDE (REDE PRÓPRLA)

$X$ SEDE DO SAMU - OLINDA

REDE DE CIRCULAÇÃO URBANA DO

MUNICIPIO DE OLINDA - PE

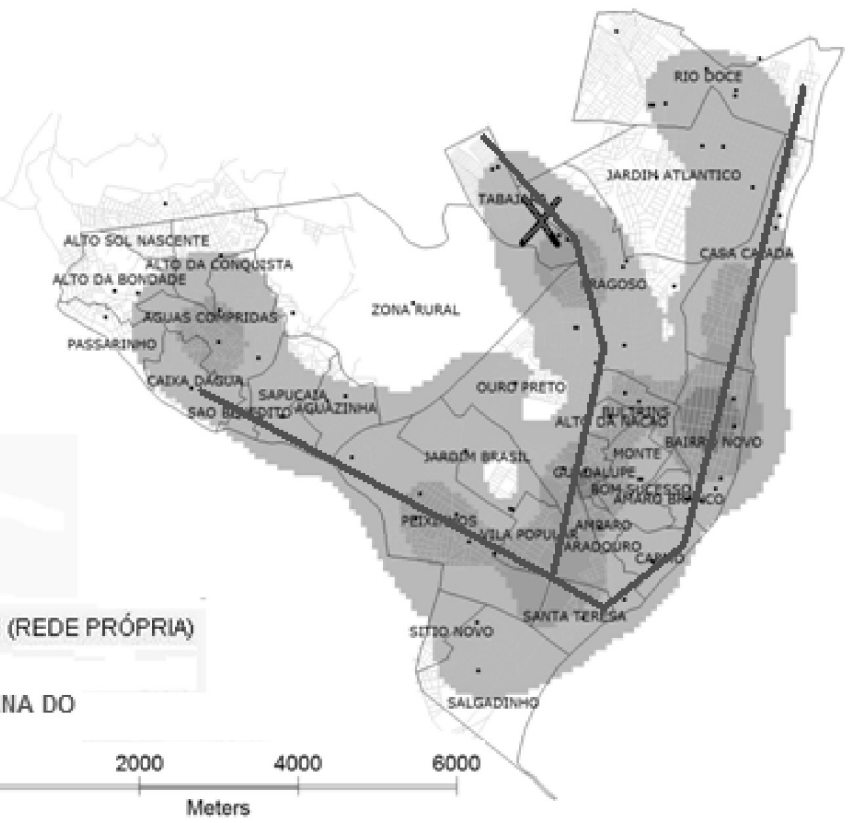

Figura 2 - Distribuição espacial das ocorrências por acidentes de transporte atendidas pelo SAMU-192-Olinda - Olinda, PE - fevereiro a junho de 2006.

Fonte: SAMU-192 Olinda, 2006

Figure 2 - Spatial distribution of traffic accidents events treated by SAMU-192/Olinda. Olinda, PE February to June 2006.

Source: SAMU-192-Olinda, 2006

Embora os carros de passeio tenham participado de $43 \%$ das ocorrências por acidentes de transporte, chama atenção o fato de $34 \%$ serem originários de motocicletas (Tabela 4).

A distribuição espacial dos acidentes de transporte, em que foi utilizado o Kernel único, é apresentada na Figura 3. Observase um foco bem expressivo no litoral, mais precisamente na região do Bairro Novo, onde se concentram dois importantes corredores viários. Não menos importantes são os focos identificados ao longo da Rodovia PE-15 (ao sul e ao norte do município); também ao sudoeste, ao longo da Av. Presidente Kennedy, no bairro de Peixinhos, bem como ao noroeste, na Estrada de Águas Compridas, no bairro de Águas Compridas. 
Tabela 3 - Motivos das ocorrências atendidas pelo SAMU-192-Olinda agrupados por capítulos da CID 10. Olinda, PE fevereiro a junho de 2006.

Table 3 - Reasons for events treated by SAMU-192-Olinda, grouped according toICD 10 chapters. Olinda, PE - February to June 2006.

\begin{tabular}{llcc}
\hline Tipos de Ocorrências & Motivo das ocorrências & $\mathrm{N}$ & $\%$ \\
\hline & Acidentes & 460 & 86,6 \\
& Acidentes de transporte & 280 & 52,7 \\
Causas Externas & Outros acidentes & 180 & 33,9 \\
& Agressões & 49 & 9,2 \\
& Demais causas externas & 22 & 4,1 \\
& Subtotal & 531 & 100,0 \\
& Ignorados & 114 & - \\
\hline \multirow{4}{*}{ Causas Clínicas } & Doenças do aparelho circulatório & 200 & 23,1 \\
& Doenças hipertensivas & 143 & 16,5 \\
& Outras doenças do aparelho circulatório & 57 & 6,6 \\
& Sintomas, sinais e achados anormais de exames clínicos e & 189 & 21,9 \\
& de laboratório, não classificados em outra parte & 119 & 13,8 \\
Total das ocorrências & Gravidez, parto e puerpério & 104 & 12,0 \\
\hline Ignorada & Transtornos mentais e comportamentais & 253 & 29,3 \\
\hline Remoções & Demais causas clínicas & 865 & 100,0
\end{tabular}

Fonte: SAMU-192-Olinda, 2006

Source: SAMU-192-Olinda, 2006

A incidência dos atendimentos por doenças do aparelho circulatório, onde se utilizou o Kernel de incidência, é apresentada na Figura 4. São observados dois focos bem delimitados a oeste, no bairro de Peixinhos e ao norte, entre os bairros de Fragoso e Ouro Preto. Outros dois focos são observados, o primeiro em parte de Águas Compridas e Sapucaia e o segundo perpassa o Sitio Histórico (Varadouro, Amparo e Carmo) até o Bairro Novo.

\section{Discussão}

A análise do perfil da demanda por serviços de atendimento pré-hospitalar móvel de urgência e emergência, aqui elaborada, foi capaz de produzir informações úteis às esferas gestoras do setor saúde do município.
A identificação de picos de atendimento segundo dias da semana pode nortear o planejamento das ações de saúde, incluindo a alocação de recursos. O aumento do volume de ocorrências por causas externas e diminuição de causas clínicas durante o final de semana exige uma equipe de resgate comprometida, treinada e experiente para lidar com este tipo de ocorrência, principalmente no que diz respeito aos acidentes de transporte. No entanto esta análise deve ser contínua, uma vez que diversos fatores podem alterar este perfil.

Fernandes $^{14}$ (2004) refere que a idade e o sexo dos pacientes merecem destaque quando analisamos as ocorrências por causas externas. Segundo a autora, estas representam a primeira causa de morte, quando consideramos a faixa etária menor 
Tabela 4 - Ocorrências atendidas pelo SAMU-192 - Olinda segundo tipo de acidente de trânsito e veículos envolvidos. Olinda, PE - fevereiro a junho de 2006.

Table 4 - Events treated by SAMU-192/Olinda, according to type of traffic accident and vehicle involved. Olinda, PE - February to June 2006.

\begin{tabular}{lccc}
\hline Variáveis & & $\mathrm{N}$ & $\%$ \\
\hline & Atropelamento & 171 & 61,1 \\
Tipo de acidente de & Colisão & 73 & 26,1 \\
trânsito & Queda no trânsito & 25 & 8,9 \\
& Capotamento & 11 & 3,9 \\
& Total & 280 & 100,0 \\
\hline & Carro de passeio & 118 & 42,6 \\
& Motocicleta & 93 & 33,6 \\
Veículo envolvido & Ônibus & 45 & 16,3 \\
& Bicicleta & 17 & 6,1 \\
& Caminhão & 4 & 1,4 \\
& Subtotal & 277 & 100,0 \\
& Ignorados & 3 & - \\
& Total & 280 & 100,0 \\
\hline
\end{tabular}

Fonte: SAMU-192-Olinda, 2006

Source: SAMU-192-Olinda, 2006

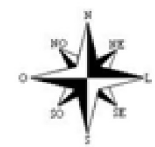

DENSIDADE DE OCORRÊNCIAS
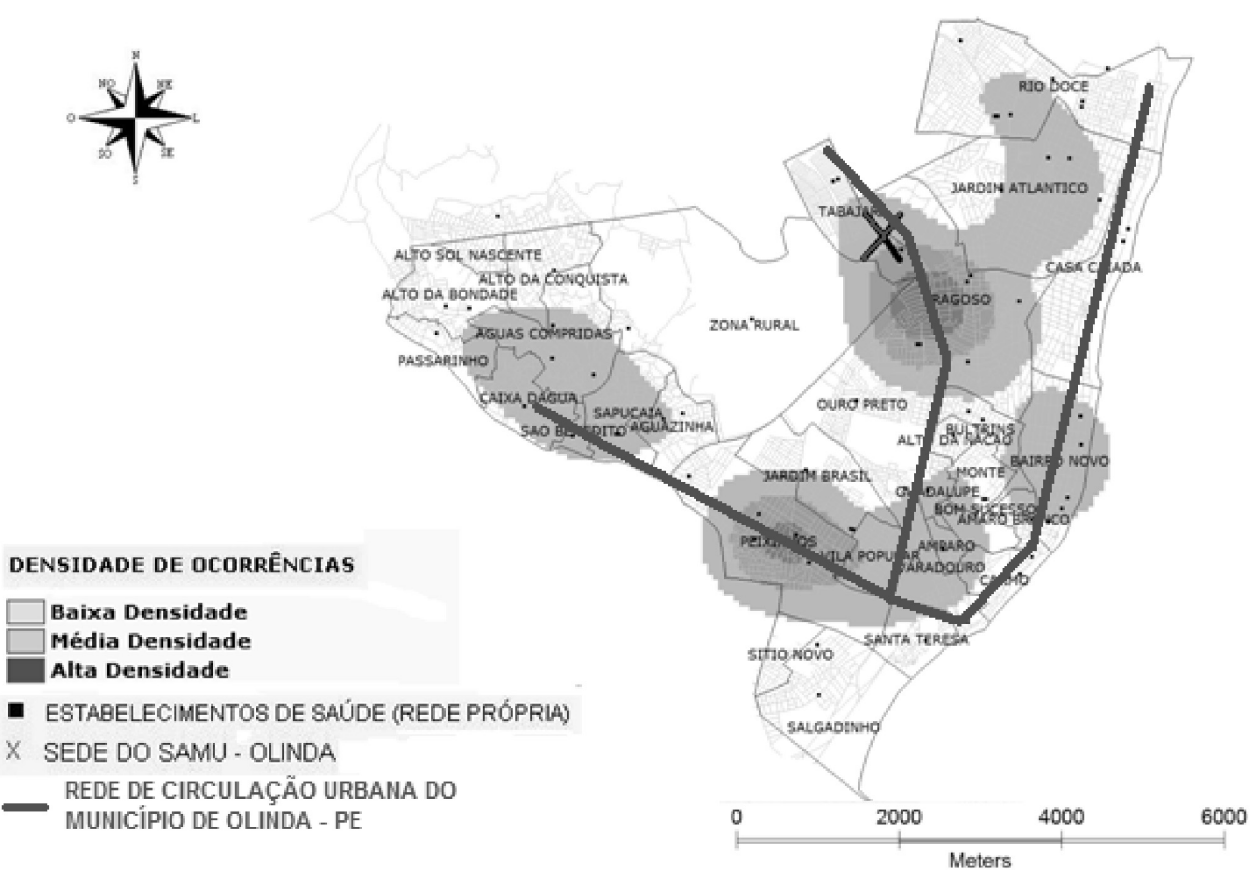

Figura 3 - Distribuição espacial da incidência de atendimentos por doenças do aparelho circulatório atendidas pelo SAMU-192-Olinda - Olinda, PE - fevereiro a junho de 2006. Fonte: SAMU-192 Olinda, 2006

Figure 3 - Spatial distribution of treatment incidence for circulatory system diseases treated by SAMU-192/Olinda. Olinda, PE - February to June 2006.

Source: SAMU-192 Olinda, 2006 
que 40 anos, representada predominantemente por pacientes do sexo masculino. Comportamento semelhante foi observado no presente estudo, o que alerta para a necessidade de ações intersetoriais de promoção da saúde e prevenção destes agravos.

Guimarães et al. ${ }^{15}$ (1995) relatam que, nos países desenvolvidos, apesar de serem os acidentes de transporte - sobretudo colisões - a primeira das causas de morte entre os jovens, a tendência tem sido decrescente devido a investimentos em medidas como obrigatoriedade do uso de cinto de segurança e diminuição do limite de velocidade.

Apesar do menor número de ocorrências em menores de 10 anos, no presente estudo observou-se uma maior incidência de atendimentos por causas clínicas em relação às causas externas nessa faixa etária. Estudos pontuam que o fato de as crianças utilizarem freqüentemente serviços de urgência $\mathrm{e}$ emergência por causas clínicas pode sinalizar o uso desses serviços em detrimento à Atenção Primária à Saúde, bem como denunciar a ausência de outros locais para atendimentos pediátricos nas proximidades da residência ${ }^{16-18}$.

Quanto à rede assistencial, o município de Olinda apresenta $70 \%$ de cobertura pela Estratégia de Saúde da Família, contando com duas unidades pré-hospitalares fixas, o Serviço de Pronto Atendimento Adulto e o Serviço de Pronto Atendimento Infantil. Conta ainda com seis policlínicas distribuídas em seus dois distritos sanitários. Devido à facilidade de acesso à cidade do Recife, é comum os munícipes de Olinda se reportarem aos serviços localizados na capital como o Hospital da Restauração (maior referência de urgência e emergência do Norte-Nordeste), Hospital Getúlio Vargas (referência para politraumatizados) e o Instituto Materno Infantil de Pernambuco (referência materno-infantil).

Face às dificuldades de oferta e organização do atendimento por parte da rede básica de serviços, a disponibilidade do SAMU-192 incita outras formas de agir frente ao adoecimento.
Kovacs et al. ${ }^{19}$ (2005), ao analisarem a acessibilidade às ações básicas de saúde de crianças atendidas em serviços de pronto socorros na cidade do Recife, identificaram como entraves entre oferta e demanda a organização do atendimento. Ressaltam ainda que vínculos entre usuários e equipe e/ou profissional e expectativas quanto à capacidade do serviço de satisfazer necessidades imediatas, além da proximidade do domicílio a algum serviço de urgência e emergência, mesmo em áreas contempladas pela Estratégia de Saúde da Família, também desempenham importante papel.

As possibilidades de entraves levantadas por Kovacs et al..$^{19}$ (2005) parecem estar de acordo não só com o comportamento dos atendimentos a menores de 10 anos, como também ao identificarmos as doenças hipertensivas, que abarcaram $16,5 \%$ do total de ocorrências por causas clínicas.

Destacaram-se as ocorrências atendidas por causas obstétricas, de parto puerpério, terceira mais freqüente dentre as causas clínicas. O SAMU-192 é um dos instrumentos da Política Nacional de Atenção Integral a Saúde da Mulher, com ênfase ao transporte e à assistência à gestante de alto risco ${ }^{20}$. Tanaka $^{21}$ (1995) refere que, em sua pesquisa realizada na Região Sul do Município de São Paulo, das 52 mortes maternas que tiveram como causas de falecimento complicações próprias associadas à gestação, ao parto e ao puerpério, $55,1 \%$ tiveram de peregrinar até a morte. De 10,2\% das demais mulheres não se tem informação suficiente para saber se passaram ou não pela peregrinação, e apenas $34,7 \%$ foram internadas prontamente. Esses dados reforçam a importância de um atendimento ágil e diligente, como deve ser o SAMU-192 e como a ocasião exige.

Fernandes ${ }^{14}$ (2004), ao levantar os motivos das solicitações do atendimento préhospitalar móvel em Ribeirão Preto, em abril de 2003, constatou que $85 \%$ das ocorrências foram por causas clínicas. Apesar de este estudo identificar uma maior proporção de atendimentos por causas clínicas (58\%), a comparação com o estudo de Ribeirão Preto mostra uma maior participação das 
ocorrências por causas externas nos atendimentos realizados na cidade de Olinda.

Chama atenção o alto percentual de dados ignorados identificados no banco de dados do serviço, o que reflete, sobretudo, a limitação da equipe no registro das informações. O responsável pelo preenchimento do formulário de ocorrências é o técnico de enfermagem que integra a equipe no momento do atendimento. É esperado um percentual de dados ignorados, pois o atendimento ao paciente, num curto espaço de tempo, deve ser priorizado. Por outro lado, ao se observar formulários com a variável sexo ignorada, por exemplo, supõe-se que a equipe deve ser orientada quanto a importância desses dados para o monitoramento do perfil de atendimentos do SAMU-192.

A distribuição dos acidentes de transporte, apresentada na Figura 3, mostra focos localizados em importantes vias de acesso do município; a região do Bairro Novo está ligada ao fluxo daqueles que se destinam às praias de Olinda e também é o segundo principal corredor de acesso do município à capital, Recife. Os focos da Rodovia PE-15 são nas duas áreas de maior circulação de pedestres: ao sul localiza-se um grande centro varejista e ao norte um dos principais terminais integrados de passageiros da Região Metropolitana do Recife. A oeste, os focos da Av. Presidente Kennedy e da Estrada de Águas Compridas podem ser justificados pelo grande movimento de pedestres sob condições inseguras e a precariedade da educação e da fiscalização do trânsito observadas. Estas informações ratificam a necessidade de um trabalho conjunto intersetorial, envolvendo diferentes órgãos da administração municipal.

A identificação de focos de ocorrências de urgências e emergências por doenças do aparelho circulatório, apresentados na Figura 4, pode apontar a melhor acessibilidade dos moradores da região aos serviços do SAMU-192-Olinda, pois no período de estudo, a Central do SAMU-192-Olinda estava localizada geograficamente próximo ao foco Fragoso/Ouro Preto. Para a análise dos focos Peixinhos e Sítio Histórico é exigida uma análise mais aprofundada com relação a estilo de vida, idade, entre outros fatores, pois, em termos de incidência de doenças do aparelho circulatório, não se esperaria a ocorrência de "clusters" espaciais.

Entretanto, o acesso viário de Olinda guarda uma particularidade: a existência de três grandes corredores viários que concentram a maior parte da circulação de veículos (ver as Figuras 2 e 3). Entre estes corredores, o acesso é limitado, sobretudo pelo relevo acidentado que compõe o município, o que pode dificultar o acesso da população destas áreas ao SAMU 192 e, por outro lado, beneficiar aquelas populações que vivem nas proximidades destas vias, como foi observado na concentração das ocorrências por doenças do aparelho circulatório.

É clara a importância do monitoramento das ocorrências atendidas pelo SAMU-192, pois o exposto sugere a necessidade não só da organização adequada de uma assistência à saúde, como também de um equacionamento dos problemas identificados, obedecendo aos princípios do SUS.

O uso da análise espacial mostra quanto é fundamental a integração entre a Secretaria Municipal de Saúde de Olinda e órgãos afins para a implantação e implementação de medidas preventivas e corretivas, com destaque para a problemática dos acidentes de transporte.

Finalmente, apesar do número significativo de ocorrências atendidas por causas externas, estão as causas clínicas, com destaque para as doenças do aparelho circulatório, responsáveis pelo maior volume de solicitações, indicando a necessidade do estabelecimento de novas estratégias de planejamento e vigilância em saúde.

\section{Agradecimentos}

Agradecemos à Tereza Cristina Miglioli (CPqAM/Fiocruz) pelo estímulo e considerações feitas neste artigo e à Secretaria de Saúde de Olinda pelo apoio no desenvolvimento desta pesquisa. 


\section{Referências}

1. Brasil. Portaria No 2048/GM de 5 de novembro de 2002. Diário Oficial da República Federativa do Brasil, Brasília, DF, 12 nov. 2002. Disponível em http://dtr2001.saude. gov.br/samu/legislacao/leg_2048.htm. [Acessado em 10 de maio de 2006].

2. Dallari SG, Pittelli SM, Pirotta WRB, Oliveira, ML. Atendimento médico de urgência na grande São Paulo. Saúde e Sociedade 2000; 10:02. Disponível em http:// www.apsp.org.br/saudesociedade/X_2/atendimento_ medico.htm. [Acessado em 27 de dezembro de 2006].

3. Brasil. Portaria No $1.863 /$ GM de 29 de setembro de 2003. Diário Oficial da República Federativa do Brasil, Brasília, DF, 6 out. 2003. Disponível em http://www.cremesp. org.br/library/modulos/legislacao/versao_impressao. php?id=3232. [Acessado em 10 de maio de 2006].

4. Brasil. Portaria No 1.864 /GM, de 29 de setembro de 2003. Diário Oficial da República Federativa do Brasil, Brasília, DF, 6 out. 2003. Disponível em: http:/ /www.cremesp. org.br/library/modulos/legislacao/versao_impressao. php?id=3232. [Acessado em 10 de maio de 2006].

5. Brasil. Ministério da Saúde. SAMU-192: O que é o $S A M U$ ? Disponível em http://www.saude.gov.br/samuprograma-nacional.htm. [Acessado em 7 de junho de 2006].

6. Freitas, RCM. O governo Lula e proteção social no Brasil: desafios e perspectivas. Rev Katál 2007;10(1): 65-74.

7. Rede Interagencial de Informações para a Saúde. Sistemas de informação geográfica e a gestão da saúde no município. Brasília; 1999.

8. Center for Disease Control and Prevention-CDC. Epiinfo versão 3.3.2. Disponível em http:/ /www.cdc.gov. [Acessado em 20 de março de 2006].

9. Brasil. Ministério da Saúde. Departamento de Informática do SUS. Informações de Saúde. Demográficas e socioeconômicas. Disponível em http:// www.datasus.gov.br. [Acessado em 15 de janeiro de 2007].

10. Brasil. Agência Nacional das Águas. Relatório de Conjuntura de Recursos Hídricos 2005. Disponível em http://www.ana.gov.br/portalestudos/conjuntura/ arquivosparadowload/nivelPais/Densidade DemograficaPorMunicipioEEstados.pdf. [Acessado em 5 de novembro de 2007].
11. Brasil. Ministério de Ciência e Tecnologia. Instituto Nacional de Pesquisas Espaciais. TerraView versão 3.1.3. Disponível em http://www.dpi.inpe.br/terraview/index. php. [Acessado em 1 de outubro de 2006].

12. Bailey TC, Gatrell AC. Interactive Spatial Analysis. $1^{\mathrm{a}}$ edição. Essex: Longman; 1995.

13. Brasil. Ministério da Saúde. Secretaria de Vigilância em Saúde. Fundação Oswaldo Cruz. Simone M. Santos e Wayner Souza (orgs.). Introdução à estatística espacial para a saúde pública. Brasília; 2006.

14. Fernandes RJ. Caracterização da atenção pré - hospitalar móvel da Secretaria de Saúde do município de Ribeirão Preto - SP [dissertação de mestrado]. Ribeirão Preto: Universidade de São Paulo; 2004.

15. Guimarães MJB, Lessa F, Regazzi AP, Aquino T, Melo N. Violência urbana em Recife: ascensão da mortalidade por causas externas 1980-1991. In: Anais do $1^{\circ}$ Congresso Brasileiro de Ciências Sociais em Saúde; 1995; Curitiba (Br). Rio de Janeiro: ABRASCO; 1995. p. 53.

16. Chatkin M, Menezes AMB, Albernaz E, Victora CG, Barros FC. Fatores de risco para consultas em pronto-socorro por crianças asmáticas no Sul do Brasil. Rev Saúde Pública 2000. 34; 491-8.

17. Carvalho MS, D’Orsi Eleonora, Prates EC, Toschi WDM, Shiraiwa T, Campos TP, et al. Demanda ambulatorial em três serviços da rede pública do município do Rio de Janeiro, Brasil. Cad Saúde Pública 1994; 10: 17-29.

18. Jacobs PC, Matos EP. Estudo exploratório dos atendimentos em unidades de emergência em Salvador - Bahia. Rev Assoc Med Bras 2005; 51: 348-53.

19. Kovacs MH, Feliciano KVO, Sarinho SW, Veras AACA. Acessibilidade às ações básicas entre crianças atendidas em serviço de pronto-socorro. J Pediatr 2005; 81: 251-8.

20. Brasil. Ministério da Saúde. Secretaria de Atenção à Saúde. Departamento de Ações Programáticas Estratégicas. Política nacional de atenção integral à saúde da mulher: plano de ação 2004-2007. Brasília; 2004.

21. Tanaka, ACD. Maternidade: dilema entre nascimento e morte. São Paulo: Hucitec; Rio de Janeiro: Abrasco; 1995.

Recebido em: 21/11/07 Versão final reapresentada em: 24/08/08 Aprovado em: 10/09/08 